\title{
Stochastic Resonance: The role of Potential Asymmetry and Non Gaussian Noises*
}

\author{
Horacio S. Wio ${ }^{\dagger}$ and Sebastián Bouzat ${ }^{\ddagger}$ \\ Comisión Nacional de Energía Atómica, \\ Centro Atómico Bariloche (CNEA) and \\ Instituto Balseiro (CNEA and UNC) \\ 8400-San Carlos de Bariloche, Argentina.
}

Received 07 December, 1998

\begin{abstract}
Within the two state theory (TST) for stochastic resonance (SR) we analize two different aspects: (a) the extension of the TST in order to include potential asymmetry (i.e.: the states show different stabilities); (b) the evaluation of transition rates for systems whose stationary distribution is non Gaussian. We apply the results of (a) to study the role of the potential symmetry for SR in bistable systems, observing that the signal-to-noise ratio increases with the symmetry of the potential of the system indicating that it is this feature that governs the optimization of the response. We apply the results of (b) to discuss SR in situations where we can assume that the noise is non Gaussian, and discuss its relation with experimental results in sensory systems.
\end{abstract}

\section{Introduction}

The phenomenon of stochastic resonance (SR) has attracted considerable interest in the last decade due, among other aspects, to its potential technological applications for optimizing the output signal-to-noise ratio (SNR) in nonlinear dynamical systems as well as its relation with some biological mechanisms. The phenomenon shows the counterintuitive role played by noise in nonlinear systems as it contributes to enhance the response of a system subject to a weak external signal. There is a wealth of papers, conference proceedings and reviews on this subject [1], Ref. [2] being the most recent one, showing the large number of applications in science and technology, ranging from paleoclimatology [3], to electronic circuits [4], lasers [5], chemical systems [6], and the connection with some situations of biological interest (noise-induced information flow in sensory neurons in living systems, the influence in ionchannel gating or in visual perception) [7]. A tendency shown in recent papers, and determined by the possible technological applications, points towards achieving an enhancement of the system response (that is: obtaining a larger output SNR) by means of the coupling of several SR units in what conforms an extended medium $[8,9,10]$.

A vast majority of studies on SR have been done analyzing a paradigmatic system: a bistable onedimensional double-well system. Among the bistable models there is one that singles out: the two-state model (TST) $[3,11]$. Such a model has proven to be extremely useful for the understanding of the SR phenomenon, offering also a simple framework to provide analytical results. Most of the studies have been carried out in the symmetric case. However, even in the earliest account of the TST [3] the possibility of asymmetry was introduced with the conclusion that the symmetric case would be the optimal one. Other authors have also (partially) analyzed this case (see references in [2]) for instance considering equal curvatures of the potential wells [12]. A more recent and related work [13] lacks a detailed analysis of the role of the symmetry while other points requiere to be clarified.

In almost all descriptions, and particularly within the TST, the transition rates between the two wells are estimated as the inverse of the mean first-passage-time. Such a passage time is evaluated using standard tech-

\footnotetext{
*Dedicated to the memory of Prof. W. Meckbach

${ }^{\dagger}$ Member of CONICET, Argentina, Electronic mail: wio@cab.cnea.edu.ar

${ }_{\ddagger}^{\ddagger}$ Fellow of CONICET, Argentina, Electronic mail: bouzat@cab.cnea.edu.ar
} 
niques $[14,15,16]$, and most specifically through the Kramers approximation [17]. In all cases the noises are assumed to be Gaussian [14, 15, 16]. However, recent papers analyze a particular class of Langevin (and its associated Fokker-Planck) equations having non Gaussian stationary distribution functions [18], opening the possibility of studying the effect of non Gaussian noises on SR. Such a work is based on the generalized thermostatistics proposed by Tsallis [19] that has been succesfully applied to a wide variety of physical systems [20].

In this contribution we start with an analysis of the asymmetrical case, extending the TST approach [3, 11], and deriving general expressions for the power spectral density (psd) and for the SNR for a general two-state system. These results are exploited in order to analyze the dependence of the system response on the noise intensity and on the degree of asymmetry for a bistable system, showing the central role played by the potential symmetry. We follow with the evaluation of transition rates (or first passage times) within the Kramers approximation for systems whose stationary distribution is non Gaussian [18]. These results are used to study the effect of such a form of noise on SR, and to compare with experiments and theoretical analysis on the sensory system of a crayfish [21]. We show that the system response at large values of the noise is better described by the present approach. Such a result strongly suggests a non Gaussian character of the noise in these systems.

\section{Case of Potential Asymmetry}

\section{A. Generalized Two State Model}

We start considering a system described by a discrete random dynamical variable $x$ that adopts two possible values: $c_{1}$ and $c_{2}$, with probabilities $n_{1,2}(t)$ respectively. Such probabilities satisfy the condition $n_{1}(t)+n_{2}(t)=1$. The master equation $[14,15,16]$ governing the evolution of $n_{1}(t)$ (and similarly for $n_{2}(t)=$ $\left.1-n_{1}(t)\right)$ is

$$
\begin{aligned}
\frac{d n_{1}}{d t}=-\frac{d n_{2}}{d t} & =W_{2}(t) n_{2}(t)-W_{1}(t) n_{1}(t) \\
& =W_{2}(t)-\left[W_{2}(t)+W_{1}(t)\right] n_{1}
\end{aligned}
$$

where the $W_{1,2}(t)$ are the transition rates out of the $x=c_{1,2}$ states.

If the system is subject (through one of its parameters) to a time dependent signal of the form $A \cos \left(\omega_{s} t\right)$, up to first order on its amplitude (assumed to be small) the transition rates may be expanded as

$$
\begin{aligned}
& W_{1}(t)=\mu_{1}-\alpha_{1} A \cos \left(\omega_{s} t\right) \\
& W_{2}(t)=\mu_{2}+\alpha_{2} A \cos \left(\omega_{s} t\right),
\end{aligned}
$$

where the constants $\mu_{1,2}$ and $\alpha_{1,2}$ depend on the detailed structure of the system under study. Here we remark that the $\mu_{i}$ 's, that are the (time independent) values of the $W_{i}$ 's without signal, are in general different from each other as a consequence of the different stability of the two states, and the same happens to the $\alpha_{i}$ 's [3]. These considerations are the main difference between our treatment and that of [11] where both $\mu_{1}=\mu_{2}$ and $\alpha_{1}=\alpha_{2}$, were assumed. Using Eq. (2) we can integrate Eq. (1) with the initial condition $x\left(t_{0}\right)=x_{0}$ and obtain the conditional probability $n_{1}\left(t \mid x_{0}, t_{0}\right)$. This result will allow us to calculate the autocorrelation function, the power spectral density (psd) and finally the SNR.

We follow the procedure of Ref. [11] to compute the $\mathrm{SNR}$, generalizing it in order to include the asymmetric case when $\mu_{1} \neq \mu_{2}$ and $\alpha_{1} \neq \alpha_{2}$. Once Eq. (1) is integrated we can calculate the correlation function $\left\langle x(t+\tau) x(t) \mid x_{0}, t_{0}\right\rangle$ as

$$
\begin{aligned}
\left\langle x(t+\tau) x(t) \mid x_{0}, t_{0}\right\rangle= & c_{1}^{2} n_{1}\left(t+\tau \mid c_{1}, t\right) n_{1}\left(t \mid x_{0}, t_{0}\right) \\
& +c_{1} c_{2} n_{1}\left(t+\tau \mid c_{2}, t\right) n_{2}\left(t \mid x_{0}, t_{0}\right)+c_{1} c_{2} n_{2}\left(t+\tau \mid c_{1}, t\right) n_{1}\left(t \mid x_{0}, t_{0}\right) \\
& +c_{2}^{2} n_{2}\left(t+\tau \mid c_{2}, t\right) n_{2}\left(t \mid x_{0}, t_{0}\right)
\end{aligned}
$$


For the $t$-averaged correlation function $C(\tau)=$ $\left\langle\lim _{t_{0} \rightarrow-\infty}\left\langle x(t+\tau) x(t) \mid x_{0}, t_{0}\right\rangle\right\rangle_{t}$, we obtain

$$
C(\tau)=R_{0}+R_{1} \exp (-\mu|\tau|)+R_{2} \cos \left(\omega_{s} \tau\right) .
$$

Here $\mu=\mu_{1}+\mu_{2}$ and the constants $R_{i}$ are given by

$$
\begin{aligned}
& R_{0}=\left(\frac{c_{2} \mu_{1}+c_{1} \mu_{2}}{\mu_{1}+\mu_{2}}\right)^{2} \\
& R_{1}=\frac{\left(c_{2}-c_{1}\right)^{2} \mu_{1} \mu_{2}}{\mu^{2}}+O\left(A^{2}\right) \\
& R_{2}=\frac{A^{2}\left(c_{1}-c_{2}\right)^{2}\left(\alpha_{2} \mu_{1}+\alpha_{1} \mu_{2}\right)^{2}}{2 \mu^{2}\left(\mu^{2}+\omega^{2}\right)} .
\end{aligned}
$$

Then, noting that $R_{0}$ is just the square of the mean value of $x$ in the absence of signal $\left(R_{0}=\left.\langle x\rangle^{2}\right|_{A=0}\right)$, we compute the t-averaged psd $\left(\langle\tilde{S}(\omega)\rangle_{t}\right)$ as the Fourier transform of $\left(C(\tau)-R_{0}\right)$. After that, we compute the one-sided t-averaged psd $(S(\omega))$, defined for $\omega>0$, as

$$
S(\omega)=\langle\tilde{S}(\omega)\rangle_{t}+\langle\tilde{S}(-\omega)\rangle_{t} .
$$

We finally get

$$
S(\omega)=4 R_{1} \frac{\mu}{\left(\mu^{2}+\omega^{2}\right)}+2 \pi R_{2} \delta\left(\omega-\omega_{s}\right) .
$$

In the one-sided $t$-averaged psd (Eq. (7)), two contributions can be distinguished: the signal output which is given by the $\delta$ function centered at the signal frequency and the broadband noise output, given by a dominant $\left(O\left(A^{0}\right)\right)$ Lorentzian term pluss some less important $\left(O\left(A^{2}\right)\right)$ terms that have been neglected.

If when calculating the power spectrum, instead of $\left(C(\tau)-R_{0}\right)$, only $C(\tau)$ is considered, an extra term $\left(4 \pi R_{0} \delta(\omega)\right)$ appears in Eq. (7). Note that a non vanishing value of $R_{0}$ can be caused either by an asymmetric choice of the values of $c_{1}$ and $c_{2}\left(c_{1} \neq-c_{2}\right)$ or by a difference in the stabilities of both states $\left(\mu_{1} \neq \mu_{2}\right)$ even when $c_{1}=-c_{2}$ is considered. If we consider the symmetric case $\left(\mu_{1}=\mu_{2} \equiv \tilde{\alpha_{0}} / 2\right.$ and $\left.\alpha_{1}=\alpha_{2} \equiv \tilde{\alpha_{1}} / 2\right)$ and also fix $c_{2}=-c_{1} \equiv c$, we recover exactly the result of [11].

For the general asymmetric case we define $R$, the $\mathrm{SNR}$, as the ratio of the strength of the output signal and the broadband noise output evaluated at the signal frequency, obtaining

$$
R=\frac{\pi R_{2}}{R_{1} \frac{2 \mu}{\left(\mu^{2}+\omega_{s}^{2}\right)}}=\frac{A^{2} \pi\left(\alpha_{2} \mu_{1}+\alpha_{1} \mu_{2}\right)^{2}}{4 \mu_{1} \mu_{2}\left(\mu_{1}+\mu_{2}\right)} .
$$

This result shows that the well known independence of the SNR on the signal frequency for small signal amplitude for symmetric systems [11] is also found to be valid when the symmetry is broken. It is worth remarking that when we consider the symmetrical case all our results reduce to those in [11].

In the case analyzed in the following subsection we will work with $R / A^{2}$ instead of $R$ and rename it: $R / A^{2} \rightarrow R$, that now will characterize the SNR independently of both the signal frequency and amplitude. Such a case corresponds to the application of this theory to study the SR of a double-well system in order to analyze the role played by the asymmetry in a simple standard example.

\section{B. Application to a Simple Bistable System}

Here we apply the theory described in the previous subsection to the following stochastic system

$$
\dot{u}(t)=-\left(u^{2}-1\right)(u+a)+S(t)+\sqrt{2} \xi(t),
$$

where $\xi(t)$ is a Gaussian white noise of zero mean and correlation $\left\langle\xi(t) \xi\left(t^{\prime}\right)\right\rangle=\eta \delta\left(t-t^{\prime}\right)$. The corresponding quartic potential is

$$
V(u)=\frac{u^{4}}{4}+\frac{a u^{3}}{3}-\frac{u^{2}}{2}-(a+S(t)) u
$$

For $S(t)=0$ it has minima at $u= \pm 1$ and a maximum at $u=-a$ and it is symmetric around $u=0$ for $a=0$. For $S(t) \neq 0$ but small, up to first order in $S(t)$, the extrema are located at

$$
\begin{gathered}
u_{1}=1+\frac{S(t)}{2(1+a)}, \quad u_{2}=-1+\frac{S(t)}{2(1-a)} \\
\text { and } u_{m}=-a-\frac{S(t)}{1-a^{2}}
\end{gathered}
$$

In Fig. 1, we depict the form of the potential for the symmetric situation $(a=0$ with $S(t)=0)$ and for an asymmetric case for two different values of the signal.

In order to apply the theory of the previous section we set $S(t)=A \cos \left(\omega_{s} t\right)$ and assume that $\left(\omega_{s}\right)^{-1}$ is large compared to the characteristic relaxation times in both wells. This allows us to approximate the system by the TST. The transition rates between the wells are given by the Kramers-like formulas 


$$
\begin{aligned}
& W_{u_{1} \rightarrow u_{2}} \equiv W_{1}=\frac{\sqrt{\left|V^{\prime \prime}\left(u_{m}\right)\right| V^{\prime \prime}\left(u_{1}\right)}}{2 \pi} \exp \left[-\frac{\left(V\left(u_{m}\right)-V\left(u_{1}\right)\right)}{\eta}\right] \\
& W_{u_{2} \rightarrow u_{1}} \equiv W_{2}=\frac{\sqrt{\left|V^{\prime \prime}\left(u_{m}\right)\right| V^{\prime \prime}\left(u_{2}\right)}}{2 \pi} \exp \left[-\frac{\left(V\left(u_{m}\right)-V\left(u_{2}\right)\right)}{\eta}\right],
\end{aligned}
$$

where $V^{\prime \prime}$ is the second derivative of $V$ with respect to $u$. The parameters $\mu_{i}$ and $\alpha_{i}$ result to be functions of $a$ and $\eta$ that can be analytically calculated as

$$
\begin{array}{lll}
\mu_{1}=\left.W_{1}\right|_{S(t)=0} & ; & \alpha_{1}=-\left.\frac{d W_{1}}{d S(t)}\right|_{S(t)=0} \\
\mu_{2}=\left.W_{2}\right|_{S(t)=0} & ; & \alpha_{2}=\left.\frac{d W_{2}}{d S(t)}\right|_{S(t)=0} .
\end{array}
$$

Then we can compute the SNR (as explained in the previous section) as a function of $a$ and the noise intensity $\eta$. The parameter $a$ characterizes the symmetry of the potential as follows: setting $a=0$ corresponds to modulating around a symmetric situation in which both states are equally stable, and $a>0$ corresponds to modulating around a situation in which the state $c_{1}$ (the well around $u_{1}$ ) is more stable than the state $c_{2}$. Finally, $a<0$ corresponds to modulating around the opposite situation where the state $c_{2}$ is the more stable one. However, as the system is invariant under the simultaneous transformations $a \rightarrow-a, u \rightarrow-u$, and $S(t) \rightarrow-S(t)$, the results of $R$ for $a$ are the same to those for $-a$ and hence, we will only consider the case $a>0$.

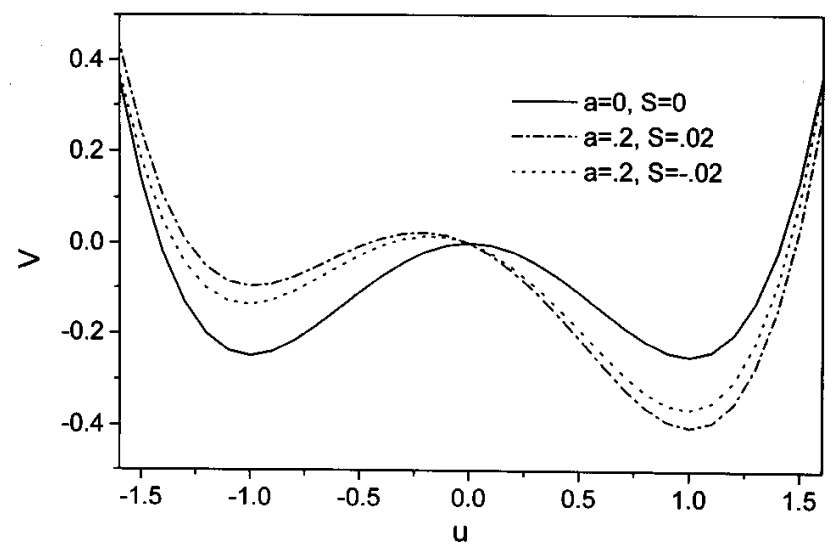

Figure 1. Potential $V(x)$ for different values of the parameters.
In Fig. 2 we show the results of $R(\eta)$ for different values of $a$. Note that each curve shows an optimum noise intensity where the SNR has a maximum; this is the typical characteristic of the SR phenomenon. Furthermore, it can be appreciated that the value of the maximum of $R$ increases with the symmetry of the system (i. e. with the proximity of $a$ to zero). Actually, for any given value of $\eta, R$ is maximized by setting $a=0$. Hence the symmetric situation is the more favorable one for the SR phenomenon.

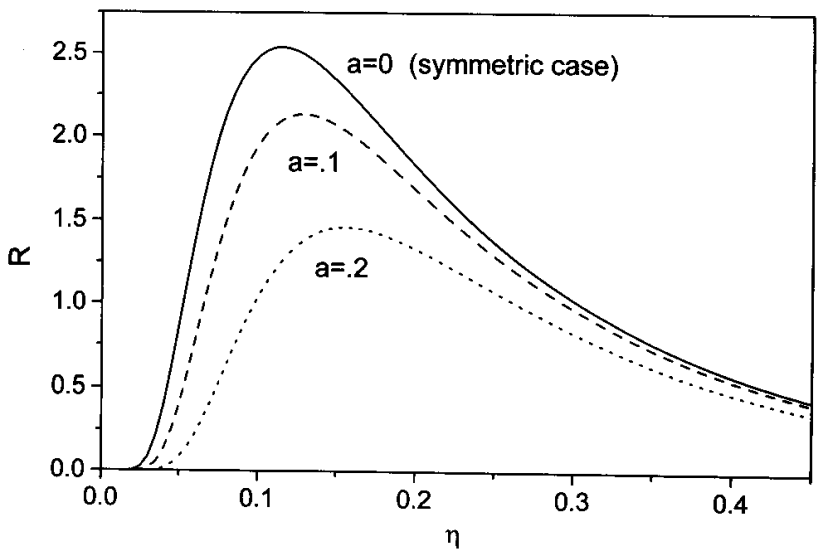

Figure 2. SNR as a function of the noise intensity for different values of the parameter $a$.

In Fig. 3 we show the value of the maximum of $R$ plotted as a function of $a$. The exact analytical expression of $R$ as a function of $a$ and $\eta$ is complicated and we will not give it here. The optimization of $R$ that occurs in the symmetric case $(a=0)$ is apparent.

This generalized TST approach has also been applied to bistable reaction-diffusion systems, exploiting the known form of the nonequilibrium potential [22]. Such results offers additional support to the above indicated role of the potential symmetry. 


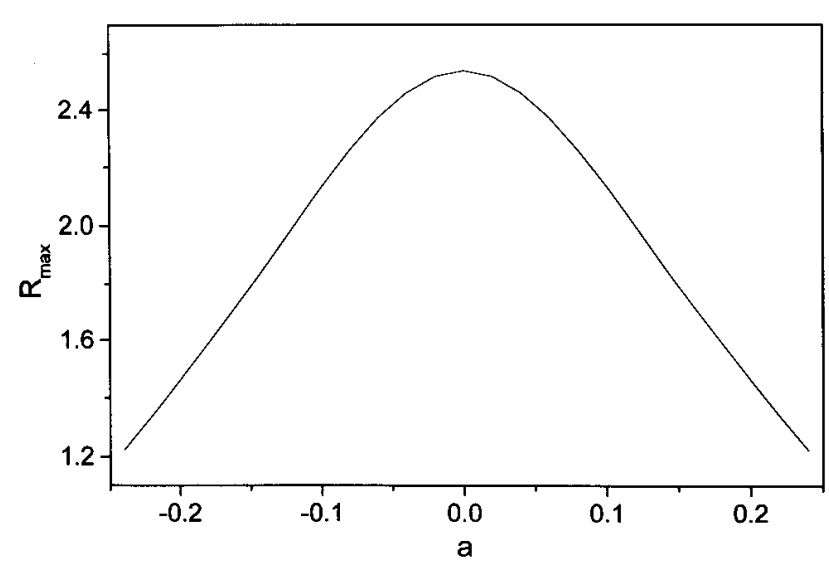

Figure 3. Maximum of $R\left(R_{\max }\right)$ as a function of $a$. The maximun of $R_{\text {max }}$ occures for $a=0$ which corresponds to the modulation around the symmetric situation.

\section{First-Passage Time and SR with non Gaussian Noises}

Traditionally, a tight connection between standard linear Fokker-Planck or Langevin equations and Gaussian (Boltzmann-Gibbs like) distributions was assumed. However, some recent papers have shown that there is an entire family of microscopic Langevin equations (with its associated Fokker-Planck equations) such that the resulting process has a Tsallis distribution [19] on the macroscopic level $[18,23,24]$. One of the possible interpretations is that the noise source is non Gaussian.

Here we present a brief account of the calculation of the transition rates (or first passage times) within the Kramers approximation for systems whose stationary distribution is non Gaussian, and the use of those results to analyze the $\mathrm{SR}$ and the effect on the SNR. A detailed account of this analysis will be presented elsewhere [25].

In Ref. [18] it was shown that a Fokker-Planck equation with constant diffusion coefficient $D$ (that measures the intensity of fluctuations)

$$
\frac{\partial}{\partial t} P(x, t)=\frac{\partial}{\partial x}\left[\frac{\partial V(x)}{\partial x} P(x, t)\right]+D \frac{\partial^{2}}{\partial x^{2}} P(x, t)
$$

with the "generalized potential"

$$
V(x)=\frac{1}{\beta(q-1)} \ln [1+\beta(q-1) U(x)],
$$

has the following stationary distribution $[18](\beta=1 / D$, with $D$ the noise intensity)

$$
\begin{aligned}
P_{s t}(x) & =N \exp (-\beta V(x)) \\
& =N[1+\beta(q-1) U(x)]^{\frac{1}{1-q}},
\end{aligned}
$$

where $N$ is a normalization factor. The results of Ref. [18] have two alternative interpretations. The obvious one corresponds to the study of diffusion in a potential given by $V(x)$, induced by a white Gaussian noise. The other possibility is to consider that we are studying diffusion in a potential given by $U(x)$ and subject to a non-Gaussian noise.

It is well known that for a potential like the one in Fig. 1, with a stationary distribution $P_{s t}(x) \sim$ $\exp [-V(z) / D]$, the first-passage time is given by $[14$, $15,16,17]$ (here we consider the symmetric case, hence $\left.a=-u_{m}=0\right)$

$$
T\left(u_{2} \rightarrow x_{0}\right)=\frac{1}{D} \int_{u_{2}}^{x_{0}} d y \exp [V(y) / D] \int_{-\infty}^{y} d z \exp [-V(z) / D] .
$$

When we replace the form of the potential given in Eq. (15), and make the integrals using the standard stepeest descent method, the first passage time adopts the form

$$
T\left(u_{2} \rightarrow 0\right)=\frac{N}{D}\left[\frac{1+\beta(q-1) U\left(u_{2}\right)}{1+\beta(q-1) U(0)}\right]^{\frac{1}{1-q}}\left[\frac{4\left(1+\beta(q-1) U\left(u_{2}\right)\right)(1+\beta(q-1) U(0))}{\beta^{2} U^{\prime \prime}\left(u_{2}\right)\left|U^{\prime \prime}(0)\right|}\right]^{1 / 2},
$$

with

$$
N=\int_{-\infty}^{\infty} d y\left[1+(q-1) y^{2}\right]^{\frac{1}{1-q}} \int_{-\sqrt{\frac{1}{1-q}}}^{\sqrt{\frac{1}{1-q}}} d z\left[1-(q-1) z^{2}\right]^{\frac{1}{q-1}}
$$

In the limit $q \rightarrow 1$ it is easy to see that $N \rightarrow \pi$, and also that $T\left(u_{2} \rightarrow 0\right)$ reduces to the known result (i.e.: Kramers formula $[17])$. 
With the previous results we are in position to evaluate the transition rates and to write the expresions for the psd $S(\omega)$ (Eq.(7)) and the SNR (Eq. (8)). The expression for $R$ results (in our case $U(0)=0$ )

$$
R \sim \frac{\pi A^{2}}{16 N D^{2}}(q+1)^{2} \sqrt{U^{\prime \prime}\left(u_{2}\right)\left|U^{\prime \prime}(0)\right|}\left[1+\frac{q-1}{D} U\left(u_{2}\right)\right]^{-\frac{1}{1-q}-\frac{1}{2}} .
$$

In the limit $q \rightarrow 1$ it reduces to the well known result [11].

However, it is still necessary to correct one drawback: the above indicated results are dependent on the energy reference level. As was disscused in [26], in order to avoid some consequences for the use of a nonextensive form of the entropy (the distributions are not invariant under uniform translations of the energy spectrum; the non-preservation of the norm; and that the first principle of thermodynamics does not preserve macroscopically the same form it has microscopically), it is requiered what the authors called the third choice for the internal energy constraint. The complication is that many quantities can only be obtained in a selfconsisting form. As within the second choice all calculations are much easier, it is better to work within it and afterwards to establish the relation between the parameters. When we consider such an approach, it becomes necessary to relate the value of the parameter $\beta$ from the third choice $(\beta)$ with that from the second one $(\tilde{\beta})$.

According to [26] we have the relation between the actual (third choice) value of $\beta$ and the "operational" (second choice) value $\tilde{\beta}$ given by

$$
\beta=\frac{\tilde{\beta}\left[\sum p_{j}^{(2)}(\tilde{\beta})^{q}\right]^{2}}{\sum p_{j}^{(2)}(\tilde{\beta})^{q}-(1-q) \tilde{\beta} U^{(2)}(\tilde{\beta})},
$$

where $p_{j}^{(2)}(\tilde{\beta})$ and $U^{(2)}(\tilde{\beta})$ are the probabilities and averaged potential evaluated within the "second choice". Clearly, for $q \rightarrow 1$ we have $\beta \rightarrow \tilde{\beta}=1 / D$.

Taking into account the indicated correction, we can calculate the SNR according to Eq. (20), and correct the values of $\beta$ according to Eq. (21). It is worth to point out that not all the values of $q$ are allowed, with some limits imposed by the conditions that the probabilities be positive definite and that the SNR do not diverge for $D \rightarrow 0$. Such conditions yield $1 \leq q \leq 5 / 3$.

At this point it becomes necessary to connect the present bistable model with the excitable one studied in Ref. [21]. In the indicated reference, the SNR is obtained via a Fourier expansion of the time periodic rate $\alpha(t)$, and a Kramers-type (time dependent) formula to evaluate the first few coefficients. The result is an expression for the SNR similar to the one arising from a TST approach [11]. The only difference is a factor $2 \mathrm{in}$ the denominator of the exponent (see Eq. (10) in [21]). Hence, it is clear that we can use our results above as an approximation of the Kramers time and as a (simple) modelization of the SNR in the indicated situation

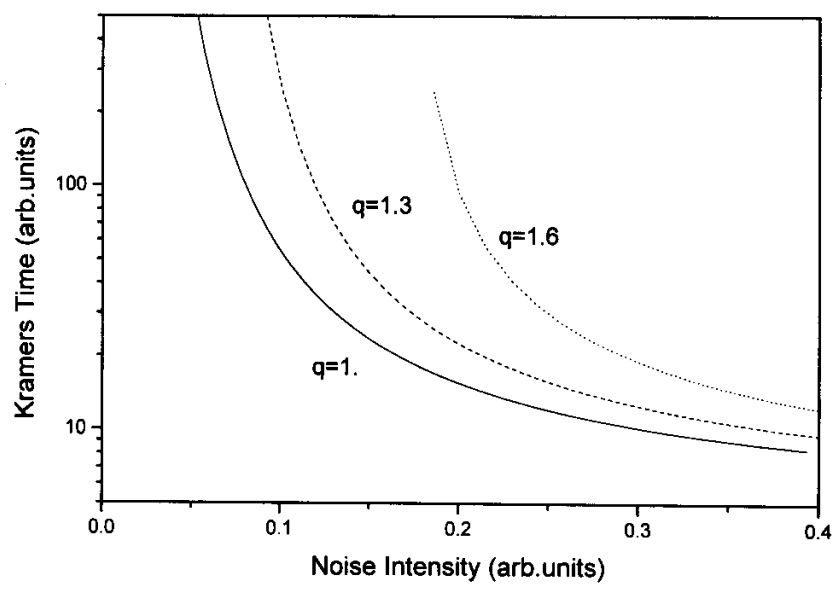

Figure 4. Kramers time (Eq. (18)) as a function of the noise intensity $D$ for different values of $q$.

In Fig. 4 we show the results for the first passage time, for different values of $q$, for a potential $U(x)$ similar to the one shown in Fig. 1 (but in the symmetric case). The general trend is an increase of $T$, particularly for low values of $D$, when $q$ increases. In Fig. 5 we show the results for the SNR as a function of $D$ for different values of $q$. The comparison of the curve with $q=1$ with those for $q>1$, clearly show an increase of the SNR for large values of $D$. When we compare this result with the curves presented in Fig. 3 of Ref. [21] it is apparent that the system's response at large values of the noise is better described by the present approach than with the simple theoretical approach used 
there. Such a result strongly suggests a non Gaussian character of the noise in these kind of systems.

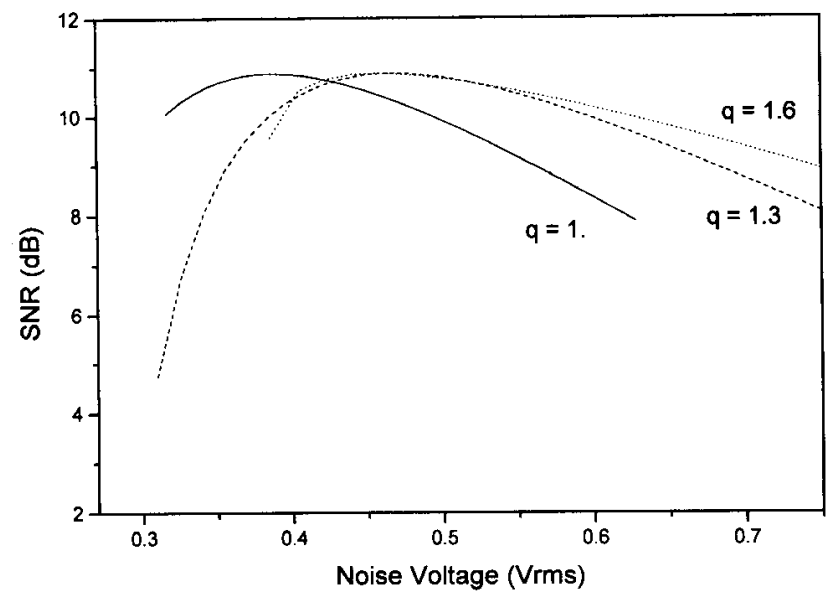

Figure 5. SNR as a function of the rms noise voltage (defined as $\sqrt{\left\langle\xi^{2}\right\rangle}=\sqrt{2 D}$ ) for different values of $q$.

\section{Conclusions}

In this contribution we have analyzed the role of potential symmetry in the SR for bistable systems without spatial extention, for the case of small signal amplitudes. We have extended the TST of SR $[3,11]$ in order to include situations with potential asymmetry. We have shown that the results for SNR for general asymmetric systems are independent of the signal frequency. An important aspect of our treatment is that we have found a way to effectively reduce any bistable system to a discrete two-state one, however for the case of small signal amplitudes. We have used this extended theory to analyze the SR of a simple system: a double-well potential, and have found that the symmetric situation is the optimal one in order to improve the SNR. It is worth mentioning that we have obtained essentialy the same results in other different bistable systems, particualrly in spatially extended systems. Furthermore this behavior seems to be independent of the way in which the signal is introduced in the system [22].

Here we want to remark that these results differs from those found in [13]. In one hand our result for the SNR shows that the well known independence of the SNR on the signal frequency for a small signal amplitude for symmetric systems [11] is also found to be valid when the symmetry is broken. On the other hand, in
$[10,22]$ it was shown that the FitzHugh-Nagumo model, in the bistable regime, has a (nonequilibrium) potential (although the system is nongradient), indicating that the claim in [13] of studying a "nonpotential system" is wrong.

The second aspect we have analyzed, is related with the possible non Gaussian character of the noise source. In general, the transition rates between the two wells are estimated as the inverse of the Kramers decay time [17], evaluated with the assumption that the noise is Gaussiann [14, 15, 16]. We have used recent results, where a particular class of Langevin equations having non Gaussian stationary distribution functions were studied [18]. The evaluation of the decay time within a Kramers approximation allows us to obtain all the relevant quantities to disscus SR within such a framework: the correlation functions, the psd, and the SNR. All those results reduce to the known ones as the "Tsallis parameter" $[19,20] q \rightarrow 1$. The results for the SNR as a function of the noise intensity $D$ clearly indicates a marked influence of the value of $q$. The present result offers a better description of the SNR for large values of $D$ than the usual one when compared with experimetal results [21], indicating a possible non Gaussian behaviour of the noises. The lack of agreement for low noise intensity can be caused (as argued in [21]) to extra noise contributions due to the spontaneous firing of the neuron.

Finally, we want to remark that the study of both aspects, in addition to the analysis of their influence in SR, have a larger relevance than pure theoretical speculations. For instance, bistable asymmetric situations provide the appropriate framework for describing SR in voltage-dependent ion channels, as proposed in [7]. In those systems, the conducting state is associated to a higher-energy well than the non-conducting one. Also the experiments on sensory systems like those in [21] and related ones, show the need to go beyond the standard approaches in order to obtain better descriptions of the experimental data.

Acknowledgments: The authors thank C. Tsallis, G. Nicolis and M. Kuperman for useful discussions and V. 
Grunfeld for a revision of the manuscript. Support from CONICET, through grant PIP Nro.4593/96, ANPCyT, through grant PICT 97 Nr.03-00000-00988, and CEB, Bariloche, are also acknowledged.

\section{References}

[1] Proc. NATO Adv. Res. Work. on Stochastic Resonance in Physics and Biology, F. Moss, et al., eds., J. Stat. Phys. 70 No. 1/2 (1993); Proc. 2nd. Int. Work. on Fluctuations in Physics and Biology, A. Bulsara et al, eds., Nuovo Cim. D 17 (1995).

[2] L. Gammaitoni, P. Hänggi, P. Jung and F. Marchesoni, Rev. Mod. Phys. 70223 (1988).

[3] C. Nicolis, Tellus 34, 1 (1982).

[4] S. Fauve and F. Heslot, Phys. Lett. A 97, 5 (1983); R.N. Mantegna and B. Spagnolo, Phys. Rev. E 49, R1792 (1994); V.S. Anishchenko,M.A. Safonova and L.O. Chua, Int. J. Bifurcation and Chaos Appli. Sci. Eng. 4, 441 (1994).

[5] J.M. Iannelli, A. Yariv, T.R. Chen and Y.H. Zhuang, Appl. Phys. Lett. 65, 1983 (1994); A. Simon and A. Libchaber, Phys. Rev. Lett. 69, 3375 (1992).

[6] A. Guderian, G. Dechert, K. Zeyer and F. Schneider; J. Phys. Chem. 100, 4437 (1996); A. Förster, M. Merget and F. Schneider; J. Phys. Chem. 100, 4442 (1996); W. Hohmann, J. Müller and F. W. Schneider; J. Phys. Chem. 100, 5388 (1996); V. Petrov, Q.Ouyang and H.L. Swinney, Nature 388, 655 (1997); and P. De Kepper and S. Müller, private communication.

[7] J. K. Douglas, L. Wilkens, E. Pantazelou \& F. Moss, Nature 365, 337-340 (1993); H.A. Braun, H. Wissing, K. Schäfer \& M.C. Hirsch, Nature 367, 270-273 (1994); I.L. Kruglikov \& H. Dertinger, Bioelectromag. 15, 539547 (1994); F. Moss \& X. Pei, Nature 376, 211-212 (1995); J. J. Collins, C.C. Chow \& T.T. Imhoff, Nature 376, 236-238 (1995); S. M. Bezrukov \& I. Vodyanoy, Nature 378, 362-364 (1995); J. J. Collins, T.T. Imhoff \& P. Grigg, Nature 383, 770-770 (1996); B.J. Gluckman, T.J. Netoff, E.J. Neel, W.L. Ditto, M.L. Spano \& S.J. Schiff, Phys. Rev. Lett. 77, 4098-4101 (1996); S. M. Bezrukov \& I. Vodyanoy, Nature 385, 319-321 (1997); R.D. Astumian, R.K. Adair \& J.C. Weaver, Nature 388, 632-633 (1997). E. Simonotto, M. Riani, C. Seife, M. Roberts, J. Twitty \& F. Moss, Phys. Rev. Lett. 78, 1186-1189 (1997); P.C. Gailey, A. Neiman, J.J. Collins \& F. Moss, Phys. Rev. Lett. 79, 4701-4704 (1997).

[8] A. Bulsara and G. Schmera, Phys. Rev. E 47, 3734 (1993); P. Jung, U. Behn, E. Pantazelou and F. Moss, Phys. Rev. A 46, R1709 (1992); Jung and Mayer-Kress, Phys. Rev. Lett. 74, 208 (1995); J.F. Lindner, B.K. Meadows, W.L. Ditto, M.E. Inchiosa and A. Bulsara, Phys. Rev. Lett. 75, 3 (1995), and Phys. Rev. E 53, 2081 (1996); F. Marchesoni, L. Gammaitoni and A.R. Bulsara; Phys. Rev. Lett. 76, 2609 (1996); J. Vilar and J. Rubi; Phys. Rev. Lett. 78, 886 (1997).
[9] H. S. Wio, Phys. Rev. E 54, R3045 (1996); H. S. Wio and F. Castelpoggi, Proc. Conf. UPoN'96, C.R.Doering, L.B.Kiss and M.Schlesinger Eds. (World Scientific, Singapore, 1997), pg. 229; F. Castelpoggi and H.S. Wio, Europhysics Letters 38, 91 (1997); F. Castelpoggi and H. S. Wio, Phys. Rev. E 57, 5112 (1998); M. Kuperman, H.S. Wio, G. Izús and R. Deza, Phys. Rev. E 57, 5122 (1998); M. Kuperman, H.S. Wio, G. Izús, R. Deza and F. Castelpoggi, Physica A 257, 275 (1998).

[10] S. Bouzat and H. S. Wio, submitted to Physica A (1998); S. Bouzat and H. S. Wio, Phys. Lett. A (1998) in press.

[11] B. McNamara and K. Wiesenfeld, Phys. Rev. A 39, 4854 (1989).

[12] P. Jüng and R. Bartussek, in Fluctuations and Order: The New Synthesis, edited by M. Millones (Springer, New York, Berlin), pg. 35.

[13] T. Alarcón, A. Pérez-Madrid and J.M. Rubí, Phys. Rev. E 57, 4979 (1998).

[14] C. W. Gardiner; Handbook of Stochastic Methods, 2nd Ed. (Springer-Verlag, Berlin, 1985).

[15] N. van Kampen; Stochastic Processes in Physics and Chemistry, (North Holland, 1982).

[16] H. S. Wio, An Introduction to Stochastic Processes and Nonequilibrium Statistical Physics (World Scientific, Singapore, 1994).

[17] P. Hänggi, P. Talkner and M. Borkovec, Rev. Mod. Phys, 62, 251 (1990).

[18] L. Borland, Phys. Lett. A 245, 67 (1998); L. Borland Phys. Rev. E 57, 6634 (1998).

[19] C. Tsallis, J. Stat. Phys 52, 479 (1988); E. M. F. Curado and C. Tsallis, J. Phys. A 24, L69 (1991); ibidem 24, 3187 (1991), ibidem 25, 1019 (1992).

[20] A.R. Plastino and A. Plastino, Phys. Lett. A 174, 384 (1993); A.R. Plastino and A. Plastino, Physica A 222, 347 (1995); D.H. Zanette and P.A. Alemany, Phys. Rev. Lett. 75, 366 (1995); B.M. Boghosian, Phys. Rev. E 53, 4754 (1996); C. Tsallis and D.J. Bukman, Phys. Rev. E 54, R2197 (1996); V.H. Hamity and D.E. Barraco, Phys. Rev. Lett. 76, 4664 (1996); A.K. Rajagopal, Phys. Rev. Lett. 76, 3469 (1996); C. Tsallis and A.M.C. de Souza, Phys. Lett. A 235, 444 (1997).

[21] K. Wiesenfeld, D. Pierson, E. Pantazelou, Ch. Dames and F. Moss, Phys. Rev. Lett. 72, 2125 (1994).

[22] S. Bouzat and H. S. Wio, Stochastic resonance in extended bistable systems: the role of potential symmetry, submitted (1998).

[23] D.A. Stariolo, Phys. Lett. A 185, 262 (1994).

[24] G. Kaniadakis and P. Quarati, Physica A 237, 229 (1997).

[25] H. S. Wio and C. Tsallis, Decay Times and Stochastic Resonance Within A Non extensive Thermostatistical Framework, in preparation.

[26] C. Tsallis, R.S. Mendes and A.R. Plastino, Physica A 261, 534 (1998). 